\title{
Determinantes sociodemográficos da falta de prontidão para atividade física em adultos quilombolas
}

\author{
Sociodemographic determinants of lack of readiness for physical activity in \\ quilombolas (inhabitants of black communities) adults
}

\begin{abstract}
Deyvis Nascimento Rodrigues ${ }^{1}$, Ricardo Franklin de Freitas Mussi ${ }^{2 *}$, Claudio Bispo de Almeida ${ }^{3}$, Edio Luiz Petroski ${ }^{4}$, Ferdinando Oliveira Carvalho ${ }^{5}$

${ }^{1}$ Mestre em Educação Física (Universidade Federal do Vale do São Francisco; ${ }^{2}$ Mestre em Saúde Coletiva (UEFS/BA). Doutor em Educação Física (UFSC/SC); ${ }^{3}$ Graduado em Educação Física (UCSal). Mestre em Educação Física (UFSC); ${ }^{4}$ Graduação em Educação Física pela Universidade Federal de Santa Catarina. Mestrado em Educação Física pela Universidade Federal de Santa Maria. Doutorado;

${ }^{5}$ Doutorado em Educação Física na Universidade Católica de Brasília (UCB-DF)
\end{abstract}

\begin{abstract}
Resumo
Objetivo: analisar os determinantes sociodemográficos da falta de prontidão para atividade física (FPAF) em adultos quilombolas. Metodologia: trata-se de um estudo transversal com amostra representativa de residentes em quilombos de região geográfica baiana. Os dados foram obtidos por meio de entrevista com uso de formulário padronizado sobre características sociodemográficas e a FPAF. Utilizou-se qui-quadrado de Pearson para análise de associação e a regressão de Poisson para estimar razões de prevalência, ambas considerando significância estatística para $p<0,05$. Resultados: a amostra apresentou predomínio do sexo feminino $(61,2 \%)$ e idade média de 45,0+17,0 anos. A FPAF foi prevalente em 62,9\% (IC95\%: 59,6: 66,2) dos quilombolas. Os problemas ostearticulares que pioram com atividade física $(36,2 \%)$ foram os principais problemas indicados como impedimento para a inserção imediata em programas de AF. A FPAF se associou independentemente com sexo e grupo etário. Conclusão: ser do sexo feminino e ter idade $\geq 40$ anos aumentam a probabilidade para a FPAF em adultos quilombolas.

Palavras-chave: Grupo com Ancestrais do Continente Africano. Atividade física. Inquéritos Epidemiológicos.
\end{abstract}

\begin{abstract}
Objective: to analyze the sociodemographic determinants of physical activity readiness (PAR) in quilombolas adults. Methodology: this is a cross-sectional study with a representative sample of residents in quilombos of the Bahian geographical region. Data were obtained through interviews using a standardized form on sociodemographic characteristics and PAR. Pearson's chi-square was used for association analysis and Poisson regression to estimate prevalence ratios, both considering statistical significance for $p<0.05$. Results: the sample had a predominance of females (61.2\%) and a mean age of $45.0 \pm 17.0$ years. PAR was prevalent in $62.9 \%(95 \%$ Cl: 59.6:66.2) of quilombolas. Ostearticular problems that worsen with physical activity (36.2\%) were the main problems indicated as impediment to immediate insertion in PA programs. PAR was independently associated with gender and age group. Conclusion: Being female and $>40$ years of age increases the likelihood of PAR in quilombolas adults.

Keywords: African Continental Ancestry Group. Physical Activity. Health Survey.
\end{abstract}

\section{INTRODUÇÃO}

A prática regular de atividade física (AF) é um importante elemento para a promoção e manutenção da saúde (PERDERSEN; SALTIN, 2015). Baixos níveis de AF impactam significativamente na elevação dos índices de mortalidade (SCHMIDT et al., 2011). No entanto, a modernidade caracteriza-se pela diminuição da quantidade de AF nos mais diferentes segmentos populacionais. Estudos apontaram que o baixo nível de AF apresenta-se como epidemia mundial (HALLAL et al., 2012) atingindo

Correspondente/Corresponding: *Ricardo Franklin de Freitas Mussi Universidade do Estado da Bahia, Departamento de Ciências Humanas - End: Rua J. J. Seabra no158 - Bairro Estação - Jacobina - Ba - CEP: 44700-000 - Telefax: (74)3621-3337.-E-mail: rimussi@yahoo.com.br aproximadamente metade da população brasileira (MIELKE et al., 2015). Esse quadro epidemiológico indica risco aumentado para o adoecimento entre aqueles pouco ativos fisicamente.

Uma considerável parcela da população brasileira reside em comunidades quilombolas, caracteristicamente rurais, de ancestralidade negra e resistente à opressão histórica (BRASIL, 2015). Os quilombos contemporâneos são constituídos por descendentes dos negros que foram escravizados, mantendo laços de parentesco e praticantes de cultura agrícola de subsistência (MUSSI; PETROSKI, 2019), sem obrigatoriedade de ascendência daqueles que conseguiram fugir da escravidão.

Neste perfil populacional também foi descrita baixa prática de AF (BEZERRA et al., 2015; MUSSI et al., 2015), 
o que, somada à presença de vulnerabilidade socioeconômica, pode agravar a situação de saúde em diversas dessas comunidades, podendo a falta de prontidão, e seus determinantes sociodemográficos, serem considerados como fatores agravantes para o adoecimento.

Esta conjuntura epidemiológica negativa exige o desenvolvimento de ações promotoras de AF. Mas, são necessários cuidados, que ampliem os benefícios e minimizem os riscos da inserção do sujeito em programas de AF. Neste sentido, a avaliação pré-participação deve em primeira instância identificar a suspeita de fatores de risco incompatíveis com a realização de AF (GHORAYEB et al., 2019). No entanto, não foram identificadas informações na literatura que indicassem a potencialidade dos riscos à saúde advindas da participação dos quilombolas em AF regulares.

Diante da carência de informações sobre os potenciais riscos relativos ao início da prática regular de AF em populações negras, especialmente aquelas residentes em áreas rurais, a presente investigação pretende analisar os determinantes sociodemográficos da falta de prontidão para atividade física (FPAF) em adultos quilombolas.

\section{METODOLOGIA}

A microrregião geográfica de Guanambi/Bahia, contava com 42 quilombos certificados pela Fundação Palmares (BRASIL, 2016) no ano de 2016, distribuídos em 10 municípios, que compuseram o campo empírico.

Diante da indisponibilidade de informações oficiais prévias relativas à quantidade de moradores das comunidades, a população foi estimada considerando 80 famílias por quilombo (BRASIL, 2010), com dois adultos ( $\geq 18$ anos) por família, totalizando 6720 adultos.

O cálculo amostral adotou prevalência desconhecida para os desfechos, nível de confiança de $95 \%$, erro amostral tolerável de cinco pontos percentuais, efeito do delineamento de 1,5 (conglomerado em um estágio), acréscimo de $30 \%$ para recusas e $20 \%$ para perdas e confundimento (LUIZ; MAGNANINI, 2000), determinando amostra mínima de 818 sujeitos. No entanto, 1025 adultos residentes nos quilombos integraram o estudo, com $17,07 \%$ de recusas, definindo amostra final de 850 participantes, os quais aceitaram por meio da assinatura ou fornecimento da impressão digital no Termo de Consentimento Livre e Esclarecido individual participar.

$O$ desenho amostral foi desenvolvido em duas etapas: sorteio das comunidades (conglomerado), seguida de coleta censitária. Inicialmente foi realizado sorteio aleatório dos quilombos. Por meio das respectivas associações de moradores, 14 unidades sorteadas permitiram visitações para a realização da pesquisa, três recusaram participação.

Aqueles com deficiência cognitiva ou de comunicação independente foram excluídos das entrevistas. Acamados, amputados, engessados, grávidas e nutrizes com menos de seis meses foram excluídos das medidas antropo- métricas. As perdas foram definidas pela não realização de alguma medida, exame ou ausência de resposta de alguma questão da entrevista.

Durante as visitações para coleta de dados foram realizadas entrevistas face-a-face, desenvolvidas por equipe treinada para o desenvolvimento das atividades, composta por profissionais graduados e ou acadêmicos. As coletas foram realizadas em sistema de mutirão, durante os finais de semana e feriados.

A variável de desfecho é a FPAF, determinada pelo Physical Activity Readiness Questionnaire (PAR-Q), instrumento composto por sete perguntas diretas, que detecta sujeitos que devem ser encaminhados previamente à participação em programas de $\mathrm{AF}$, para revisão clínica e tratamento de problemas que podem ser agravados pela AF (SHEPHARD; BAILEY; MIRWALD, 1991). Neste sentido, conforme indicado no instrumento, é considerado(a) sem prontidão para AF aquele(a) que responder positivamente, mesmo que momentaneamente, um ou mais dos sete questionamentos, que representam contraindicação para a prática $A F$, independentemente da intensidade em que estiver presente.

As variáveis sociodemográficas investigadas foram: sexo (feminino, masculino), situação conjugal (com e sem cônjuge), grupo etário ( $<40$ anos, $\geq 40$ anos), escolaridade ( $\leq 4^{a}$ série, $>4^{a}$ série), situação laboral (sem remuneração, com remuneração).

Os dados foram analisados descritivamente (frequência relativa e absoluta). Aplicou-se o teste qui-quadrado de Pearson, com significância de 5,0\% ( $p<0,05)$, para verificar associação entre as questões do PAR-Q conforme o sexo. A análise inferencial de associação do desfecho (FPAF) com as variáveis independentes (sociodemográficas) consistiu na estimação das razões de prevalências (RP) a partir da regressão de Poisson (brutas e ajustadas), com variância robusta. Aquelas variáveis que apresentaram $p$-valor $<0,20$ na análise bruta foram incluídas no modelo para análise múltipla. As variáveis que apresentaram $p$ -valor $<0,05$ no nível permaneceram no modelo saturado. As magnitudes das associações foram estimadas pelo intervalo de confiança de $95 \%$. Todas as análises foram realizadas no programa The Statistical Package for Social Sciences, versão 22.0.

Esta análise é recorte do estudo intitulado "Perfil Epidemiológico dos Quilombolas baianos" autorizado pelo Comitê de Ética em Pesquisa com Seres Humanos da Universidade do Estado da Bahia (CEP/UNEB), sob parecer $\mathrm{n}^{0} 1.386 .019 / 2016$, desenvolvido entre fevereiro e novembro de 2016 .

\section{RESULTADOS}

Os 850 participantes apresentaram idade média de $45,0 \pm 17,0$ anos e $61,2 \%$ eram do sexo feminino. Observou-se que a FPAF foi prevalente em 62,9\% (IC95\%: 59,6: 66,2) dos participantes, com uma média de 1,26 (IC95\%: 1,17: $1,35)$ repostas positivas às perguntas do PAR-Q. 
Dentre as condições que impedem a inserção imediata em programas de AF investigadas pelo PAR-Q, 36,2\% dos entrevistados indicaram problemas osteoarticulares (problemas ósseos, articulares ou de coluna) que pioram com AF (PAR-Q5), 24,6\% citaram desconforto no peito durante a prática de AF (PAR-Q2) e 22,1\% afirmaram ter prescrição para uso de medicamentos para pressão arterial ou problemas cardíacos (PAR-Q6), sendo as três questões limitantes mais citadas (Tabela 1).

A análise dos fatores investigados pelo PAR-Q indicou que ser do sexo feminino se associa significativamente com a perda de equilíbrio por tonturas ou já ter ficado inconsciente (PAR-Q4) (Tabela 1).

Tabela 1-Prevalência dos indicadores de prontidão de atividade física em adultos quilombolas. Bahia, Nordeste, Brasil, 2016. $(n=850)$.

\begin{tabular}{|c|c|c|c|c|c|c|c|}
\hline \multirow{3}{*}{$\begin{array}{c}\text { Componente } \\
\text { da SM }\end{array}$} & \multicolumn{4}{|c|}{ Sexo } & \multirow[b]{3}{*}{ p-valor ${ }^{*}$} & \multirow{2}{*}{\multicolumn{2}{|c|}{ Total }} \\
\hline & \multicolumn{2}{|c|}{ Feminino } & \multicolumn{2}{|c|}{ Masculino } & & & \\
\hline & $\mathrm{N}$ & $\%$ & $N$ & $\%$ & & $\mathrm{~N}$ & $\%$ \\
\hline Parq-1 & & & & & 0,426 & & \\
\hline Não & 486 & $61,4 \%$ & 305 & $38,6 \%$ & & 791 & $96,0 \%$ \\
\hline Sim & 18 & $54,5 \%$ & 15 & $45,5 \%$ & & 33 & $4,0 \%$ \\
\hline Parq-2 & & & & & 0,072 & & \\
\hline Não & 369 & $59,4 \%$ & 252 & $40,6 \%$ & & 621 & $75,4 \%$ \\
\hline Sim & 135 & $66,5 \%$ & 68 & $33,5 \%$ & & 203 & $24,6 \%$ \\
\hline Parq-3 & & & & & 0,210 & & \\
\hline Não & 408 & $60,2 \%$ & 270 & $39,8 \%$ & & 678 & $82,3 \%$ \\
\hline Sim & 96 & $65,8 \%$ & 50 & $34,2 \%$ & & 146 & $17,7 \%$ \\
\hline Parq-4 & & & & & 0,019 & & \\
\hline Não & 408 & $59,4 \%$ & 279 & $40,6 \%$ & & 687 & $83,4 \%$ \\
\hline Sim & 96 & $70,1 \%$ & 41 & $29,9 \%$ & & 137 & $16,5 \%$ \\
\hline Parq-5 & & & & & 0,058 & & \\
\hline Não & 309 & $58,7 \%$ & 217 & $41,3 \%$ & & 526 & $63,8 \%$ \\
\hline Sim & 195 & $65,4 \%$ & 103 & $34,6 \%$ & & 298 & $36,2 \%$ \\
\hline Parq-6 & & & & & 0,135 & & \\
\hline Não & 384 & $59,8 \%$ & 258 & $40,2 \%$ & & 642 & $77,9 \%$ \\
\hline Sim & 120 & $65,9 \%$ & 62 & $34,1 \%$ & & 182 & $22,1 \%$ \\
\hline Parq-7 & & & & & 0,414 & & \\
\hline Não & 479 & $60,9 \%$ & 308 & $39,1 \%$ & & 787 & $95,5 \%$ \\
\hline Sim & 24 & $67,6 \%$ & 12 & $32,4 \%$ & & 37 & $4,5 \%$ \\
\hline
\end{tabular}

Parq-1: Alguma vez foi mencionado que o(a) Sr.(a) tem algum problema cardíaco ou que só poderia fazer atividade física com recomendação médica? Parq-2: O(a) Sr.(a) sente dor ou desconforto no peito quando faz atividade física?; Parq-3: Nos últimos meses o(a) Sr. (a) tem sentido dor ou desconforto no peito mesmo sem fazer atividade física?; Parq-4: $O$ (a) Sr. (a) perde o equilibrio em virtude de tonturas ou alguma vez já ficou inconsciente? Parq-5: O(a) Sr.(a) tem problemas ósseos, articulares ou de coluna que pioram quando pratica atividade física? Parq-6: Seu médico já prescreveu medicamentos para pressão arterial ou problemas cardíacos? Parq-7: O(a) Sr.(a) tem qualquer outra razão conhecida para não praticar atividade física?

Fonte: Autoria própria
Observou-se que a FPAF se associou $(p<0,05)$ com pertencer ao sexo feminino, grupo de pessoas mais velhas, com menor escolaridade, que não ter trabalho remunerado e ter mais filhos (tabela 2).

Tabela 2 - Razão de Prevalência (RP) da falta de prontidão para atividade física e seus intervalos de confiança de 95\% (IC 95\%) segundo variáveis sociodemográficas. Bahia, Nordeste, Brasil, $2016(n=850)$.

\begin{tabular}{|c|c|c|}
\hline VARIÁVEIS & RP (IC95\%) & p-valor \\
\hline \multicolumn{3}{|l|}{ Sexo } \\
\hline Masculino & 1 & \\
\hline Feminino & $1,266(1,126: 1,423)$ & $<0,0001$ \\
\hline \multicolumn{3}{|l|}{ Situação conjugal } \\
\hline Sem cônjuge & 1 & \\
\hline Com cônjuge & $0,992(0,876: 1,122)$ & 0,893 \\
\hline \multicolumn{3}{|l|}{ Grupo etário } \\
\hline$<40$ anos & 1 & \\
\hline$\geq 40$ anos & $1,628(1,346: 1,844)$ & $<0,0001$ \\
\hline \multicolumn{3}{|l|}{ Escolaridade } \\
\hline$>5$ anos & 1 & \\
\hline$\leq 5$ anos & $1,383(1,224: 1,563)$ & $<0,0001$ \\
\hline \multicolumn{3}{|l|}{ Situação laboral } \\
\hline Com remuneração & 1 & \\
\hline Sem remuneração & $1,134(1,021: 1,259)$ & 0,019 \\
\hline \multicolumn{3}{|l|}{ Renda familiar } \\
\hline$\leq 1$ salário mínimo & 1 & \\
\hline > 1 salário mínimo & $1,019(0,890: 1,166)$ & 0,786 \\
\hline \multicolumn{3}{|l|}{ Número de filhos } \\
\hline$>4$ filhos & 1 & \\
\hline$\leq 4$ filhos & $0,780(0,705: 0,863)$ & $<0,0001$ \\
\hline \multicolumn{3}{|l|}{$\begin{array}{l}\text { Número de moradores } \\
\text { no domicílio }\end{array}$} \\
\hline$>4$ moradores & 1 & \\
\hline$\leq 4$ moradores & $0,936(0,842: 1,042)$ & 0,226 \\
\hline
\end{tabular}

RP: Razão de prevalência; IC95\%: intervalo de confiança de 95\%.

Fonte: Autoria própria

Na análise de regressão múltipla (tabela 3), permaneceram independentemente associadas a FPAF as variáveis: sexo e grupo etário. Então, a análise ajustada indica que se eleva em 1,37 vez a probabilidade das mulheres apresentarem FPAF. No mesmo sentido, há aumento de 1,70 vez na probabilidade das pessoas com idade $\geq 40$ anos apresentarem FPAF. 
Tabela 3 - Análise de regressão de Poisson múltipla dos determinantes da Falta de Prontidão para atividade física em adultos quilombolas. Bahia, Nordeste, Brasil, 2016.

\begin{tabular}{|c|c|c|c|c|}
\hline VARIÁVEIS & RP * (IC95\%) & p-valor & $\begin{array}{c}\text { RP ** } \\
\text { (IC95\%) }\end{array}$ & p-valor \\
\hline \multicolumn{5}{|l|}{ Sexo } \\
\hline Masculino & 1 & & 1 & \\
\hline Feminino & $1,356(1,206: 1,525)$ & $<0,0001$ & $1,365(1,221: 1,526)$ & $<0,0001$ \\
\hline \multicolumn{5}{|l|}{ Grupo etário } \\
\hline$<40$ anos & 1 & & 1 & \\
\hline$\geq 40$ anos & $1,633(1,405: 1,899)$ & $<0,0001$ & $1,701(1,504: 1,923)$ & $<0,0001$ \\
\hline \multicolumn{5}{|l|}{ Escolaridade } \\
\hline$>5$ anos & 1 & & & \\
\hline$\leq 5$ anos & $1,123(0,977: 1,291)$ & 0,104 & & \\
\hline \multicolumn{5}{|l|}{ Situação Laboral } \\
\hline Com remuneração & 1 & & & \\
\hline Sem remuneração & $1,096(0,988: 1,216)$ & 0,083 & & \\
\hline \multicolumn{5}{|l|}{ Número de filhos } \\
\hline$>4$ filhos & 1 & & & \\
\hline$\leq 4$ filhos & 1,037 (0,933:1,153) & 0,496 & & \\
\hline
\end{tabular}

RP: Razão de prevalência; IC95\%: intervalo de confiança de 95\%; * Regressão de Poisson ajustada inicial; ** Regressão de Poisson com as variáveis que permaneceram no modelo saturado; $a$-variáveis eliminadas na análise ajustada inicial; $b$-variáveis eliminadas no segundo nível; c-variáveis eliminadas no terceiro nível.

Fonte: Autoria própria

\section{DISCUSSÃO}

Os principais achados desta análise indicam que a FPAF afeta seis em cada dez adultos quilombolas, associada independentemente com o sexo feminino e ter idade $\geq 40$ anos. Como principais motivos para a FPAF quatro em cada 10 adultos quilombolas referiram problemas osteoarticulares, dois em 10 citaram desconforto cardíaco durante a AF, mesma proporção apontada para doenças cardiovasculares. Quanto às questões do PAR-Q, ser do sexo feminino se associa com perda de equilíbrio por tonturas ou já ter ficado inconsciente (PAR-Q4). Até o momento da finalização dessa análise não foram encontrados trabalhos que tenham analisado a FPAF em quilombolas. Neste sentido, acredita-se que este seja o primeiro estudo que investigue esse desfecho nesta população.

Mesmo reconhecendo os benefícios da prática regular de AF é fundamental que seja desenvolvida uma avaliação pré-participação, com o intuito de maximização dos seus benefícios. Neste sentido, estudos nacionais (ANDREAZZI et al., 2016) e internacionais (WARBURTON et al., 2011) têm utilizado o PAR-Q para a triagem dos sujeitos mais expostos à risco decorrente da sua inserção em programas de AF.

A FPAF identificada entre os quilombolas é maior que os $20,5 \%$ identificado em adultos australianos (DUNCAN et al., 2016), e que os 5,6\% em universitários brasileiros (LOPES et al., 2013). Observa-se que o perfil de saúde dos quilombolas está condicionado a fatores sociais, culturais, políticos, econômicos e ambientais que podem potencializar a FPAF, principalmente diante da presença de enfermidades. Além disso, a população rural brasileira, assim como os quilombolas da presente análise, está sujeita à situação recorrente de pobreza, com restrição de acesso à bens e serviços importantes para a manutenção de uma vida saudável (BRASIL. MINISTÉRIO DA SAÚDE, 2013).

A presença dos problemas osteoarticulares como principal motivo para a FPAF desta investigação resultaria, mesmo que parcialmente, do perfil laboral desta população. Nestas comunidades rurais o trabalho é caraterizado pelo esforço físico com baixo apoio tecnológico, que elevam a propensão para lesões osteoarticulares (MEZIAT FILHO; SILVA, 2011). No entanto, investigação anterior desenvolvida em comunidade quilombolas do alto sertão baiano identificou prevalência de problemas ostearticulares de aproximadamente 50\% (MUSSI; TEIXEIRA; FIGUEIREDO, 2016), portanto, bastante maior que o identificado neste levantamento regional.

Diferentemente da presente pesquisa, as doenças cardiocirculatórias são o mais prevalente indicativo da FPAF em adultos australianos (DUNCAN et al., 2016). Possivelmente, como já mencionado, as diferenças culturais, com impactos no estilo de vida explicam essa diferença.

Assim como nesse estudo, investigação com adultos brasileiros (LOPES et al., 2013), identificou as mulheres significativamente mais propensas à FPAF que os participantes do sexo masculino. Sobre esta questão, é sabido que ainda perdura na sociedade uma ideia quanto aos diferentes papeis sociais conforme o sexo. Assim, essa condição pode ser resultado de um modelo de sociedade que dificulta a participação feminina em AF, principalmente aquela relativa ao tempo livre (MUSSI et al, 2015) expondo as mulheres a situações de saúde mais desfavoráveis.

Os sujeitos com 40 ou mais anos apresentaram maior FPAF quando comparado ao grupo mais jovem. Essa as- 
sociação pode ser compreendida à partir das mudanças biológicas relacionadas ao envelhecimento, que promovem a diminuição progressiva das capacidades físicas, que pode em situação mais grave desencadear enfermidades, como as osteoarticulares (LÓPEZ-OTíN et al., 2013; MAZO et al., 2012).

A perda de equilíbrio por tonturas se associou ao sexo feminino entre os quilombolas, quadro que corrobora os achados de investigação nacional (BITTAR et al., 2013) e internacional (SCHAPPERT; NELSON, 1999). Esses problemas indicam origem de doenças vestibulares, cerebelares, proprioceptivas ou das suas vias de integração com o sistema nervoso central (PAULINO et al., 2010), mais comuns em mulheres e com reflexos negativos na prática de atividade física.

$O$ desenho de estudo transversal, desta investigação representa limitação, uma vez que não permite entendimento da relação de causalidade entre a FPAF e os preditores considerados. Outra questão que deve ser ponderada é a possibilidade de viés de compreensão e de memória dos participantes, mas, os entrevistadores foram treinados para aplicação padronizada dos questionários com o intuito de sua minimização. Ainda é importante citar a ausência de estudo para adaptação transcultural do instrumento relativo a FPAF, no entanto, sua ampla capacidade de rastreio do risco é reconhecida, inclusive pela Sociedade Brasileira de Cardiologia e pela Sociedade Brasileira de Medicina do Exercício e do Esporte (GHORAYEB et al., 2019).

Os pontos fortes desta análise versão da amostra representativa de população quilombola residente em ampla área geográfica (18 cidades); e a apresentação de informações que poderão contribuir na elaboração de ações para promoção da AF em comunidade com características étnico-culturais específicas.

\section{CONCLUSÃO}

Foi identificada que a FPAF é prevalente em seis a cada dez adultos quilombolas, mais provável entre mulheres $\mathrm{e}$ diante do envelhecimento e, tendo como principal motivo, a presença de problemas osteoarticulares. Ressalta-se que programas de atividade física e saúde devem priorizar a correção prévia das questões relacionada à FPAF e o perfil populacional mais acometido antes sua implementação, o que ampliará a possibilidade de sucesso da proposta e benefícios aos seus participantes.

A disponibilidade de informações sobre as condições de saúde em comunidades quilombolas, população exposta à vulnerabilidade socioeconômica, permitirão ações que atendam suas demandas específicas, priorizando a correção de problemas mais recorrentes.

\section{REFERÊNCIAS}

ANDREAZZI, I. M. et al. Exame pré-participação esportiva e o PAR-Q, em praticantes de academias. Rev. bras. med. esporte, São Paulo, v.22, n. 4, p.272-276, 2016.
BEZERRA, V. M. et al. Domínios de atividade física em comunidades quilombolas do sudoeste da Bahia, Brasil: estudo de base populacional. Cad. Saúde Pública, [s.l.], v.31, n.6, p.1213-1224, 2015.

BITTAR, R. S. M. et al. Estudo epidemiológico populacional da prevalência de tontura na cidade de São Paulo. Braz. j. otorhinolaryngol., São Paulo, v.79, n.6, p.688-698, 2013.

BRASIL. Programa Brasil Quilombola. Brasília: Secretaria de Políticas da Promoção da Igualdade Racial, 2015. Disponível em: http://www. seppir.gov.br/comunidades-tradicionais/programa-brasil-quilombola. Acesso em $30 \mathrm{dez} .2016$

BRASIL. Fundação Palmares. Brasília: Fundação Palmares, 2016. Disponível em: http://www.palmares.gov.br. Acesso em: 24 nov 2016.

BRASIL. Ministério da Saúde. Secretaria de Gestão Estratégica e Participativa. Departamento de Apoio à Gestão Participativa. Política Nacional de Saúde Integral das Populações do Campo e da Floresta. Brasília: Editora do Ministério da Saúde, 2013.

BRASIL. Secretaria de Políticas de Promoção da Igualdade Racial da Presidência da República (SEPPIR). Comunidades Quilombolas Brasileiras - Regularização Fundiária e Políticas Públicas. Brasília, 2010.

DUNCAN, M. J. et al. What is the impact of obtaining medical clearance to participate in a randomised controlled trial examining a physical activity intervention on the socio-demographic and risk factor profiles of included participants? Trials, London, v.17, n.580, p.1-6, 2016.

GHORAYEB, N. et al. Atualização da Diretriz em Cardiologia do Esporte e do Exercício da Sociedade Brasileira de Cardiologia e da Sociedade Brasileira de Medicina do Exercício e Esporte - 2019. Arq Bras Cardiol., v.112, n.3, p.326-368, 2019.

HALLAL, P. C. et al. Global physical activity levels: surveillance progress, pitfalls and prospects. Lancet, London, v.380, n.9838, p.247-257, 2012.

LOPES, P. R. N. R. et al. Prontidão para a prática de atividade física em estudantes participantes de um torneio universitário. R. Bras. Ci. e Mov., Taguatinga, v.21, n.1, p.132-138, 2013.

LÓPEZ-OTÍN, C. et al. The hallmarks of aging. Cell, [s.I], v.153, p.11941217, 2013.

LUIZ, R. R.; MAGNANINI, M. M. F. A lógica da determinação do tamanho da amostra em investigações epidemiológicas. Cad. saúde colet., Rio de Janeiro, v.8, n.2, p.9-28, 2000.

MAZO, G. Z. et al. aptidão física, exercícios físicos e doenças osteoarticulares em idosos. Rev. bras. ativ. fís. saúde, Londrina, v.17, n.4, p.300-306, 2012.

MEZIAT FILHO, N.; SILVA G. A. Invalidez por dor nas costas entre segurados da Previdência Social do Brasil. Rev. Saúde Pública, São Paulo, v.45, n.3, p.494-502, 2011.

MIELKE, G. I. et al. Prática de atividade física e hábito de assistir à televisão entre adultos no Brasil: Pesquisa Nacional de Saúde 2013. Epidemiol. Serv. Saúde, v.24, n.2, p.277-86, 2015.

MUSSI, R. F. F. et al. Atividades físicas praticadas no tempo livre em comunidade quilombola do alto sertão baiano. Licere, Belo Horizonte, v.18, n.1, p.157-187, 2015

MUSSI, R. F. F.; PETROSKI, E. L. Síndrome metabólica e fatores associados em quilombolas baianos, Brasil. Ciênc. saúde coletiva, v.24, n.7, p.24812490, 2019.

MUSSI, R. F. F.; TEIXEIRA, E. P.; FIGUEIREDO, A. C. M. G. Problema/Dor articular e atividade física de tempo livre em Quilombo Baiano, Brasil. Rev. Ciênc. Méd. Biol., Salvador, v.15, n.1, p.68-72, 2016. 
PAULINO, C. A. et al. Sintomas de estresse e tontura em estudantes de pós-graduação. Rev. Equilíbrio Corporal Saúde, São Paulo, v.2, n.1, p.15-26, 2010.

PERDERSEN, B. K.; SALTIN, B. Exercise as medicine - evidence for prescribing exercise as therapy in 26 different chronic diseases. Scand j. med. sci. sports, Copenhagen, v. 25, supl. 3, p,1-72, 2015.

SCHAPPERT, S. M.; NELSON, C. National ambulatory medical care survey: 1995-96 summary. Vital Health Stat. 13, Washington, n.142, 1999.

SCHMIDT, M. I. et al. Chronic non-communicable diseases in Brazil: burden and current challenges. Lancet, London, v.377, n.9781, p.19491961, 2011.

SHEPHARD, R. J.; BAILEY, D. A.; MIRWALD, R. L. The canadian home fitness test update. Sports med., Auckland, v.11, n.1, p.358, 1991.

WARBURTON, D. E. R. et al. Evidence-based risk assessment and recommendations for physical activity clearance: Consensus Document 2011. Appl. physiol. nutr. metab., Ottawa, v.36, supl 1, p. S266-S298, 2011.

Submetido em: 03/08/2019

Aceito em : 30/01/2020 